\title{
A Survey of 3D Face Recognition Methods
}

\author{
Alize Scheenstra $^{1}$, Arnout Ruifrok ${ }^{2}$, and Remco C. Veltkamp ${ }^{1}$ \\ 1 Utrecht University, Institute of Information and Computing Sciences, Padualaan \\ 14, $3584 \mathrm{CH}$ Utrecht, The Netherlands \\ alize.scheenstra@tiscali.nl, remco.veltkamp@cs.uu.nl \\ 2 Netherlands Forensic Institute, Laan van Ypenburg 6, 2497 GB Den Haag, The \\ Netherlands, \\ arnout@holmes.nl
}

\begin{abstract}
Many researches in face recognition have been dealing with the challenge of the great variability in head pose, lighting intensity and direction,facial expression, and aging. The main purpose of this overview is to describe the recent $3 \mathrm{D}$ face recognition algorithms. The last few years more and more $2 \mathrm{D}$ face recognition algorithms are improved and tested on less than perfect images. However, 3D models hold more information of the face, like surface information, that can be used for face recognition or subject discrimination. Another major advantage is that $3 \mathrm{D}$ face recognition is pose invariant. A disadvantage of most presented 3D face recognition methods is that they still treat the human face as a rigid object. This means that the methods aren't capable of handling facial expressions. Although 2D face recognition still seems to outperform the $3 \mathrm{D}$ face recognition methods, it is expected that this will change in the near future.
\end{abstract}

\section{Introduction}

One of the earliest face recognition methods was presented in 1966 by Bledsoe [1]. In one of his papers [2], Bledsoe described the difficulties of the face recognition problem:

"This recognition problem is made difficult by the great variability in head rotation and tilt, lighting intensity and angle, facial expression, aging, etc. Some other attempts at facial recognition by machine have allowed for little or no variability in these quantities. Yet the method of correlation (or pattern matching) of unprocessed optical data, which is often used by some researchers, is certain to fail in cases where the variability is great. In particular, the correlation is very low between two pictures of the same person with two different head rotations."

Since that time many researches have been dealing with this subject and have been trying to find an optimal face recognition method. The main purpose of this overview is to describe the recent face recognition algorithms on still images. Previous face recognition surveys were presented by Samal and Iyengar [3], 
Chellappa et al. [4] and Zhao et al. [5]. However, they all are primarily focussed on 2D face recognition. In the Vendor Test 2002 the performance of different commercial face recognition methods were compared [6]. Most commercial face recognition systems use one or more algorithms as presented in the literature. However, all systems conceal which algorithms are used in their application. Therefore, commercial systems are excluded in this survey. The last few years 3D facial models can be more easily acquired since the acquisition techniques have improved. Therefore, some face recognition methods originally developed for $2 \mathrm{D}$ face recognition have been extended for 3-dimensional purposes. Using $3 \mathrm{D}$ models one can deal with one main problem in 2D face recognition: the influence of the pose of the head. Also the surface curvature of the head can now be used to describe a face. A recent survey of 3D face recognition was recently presented by Bowyer [10]. Since that time new results with respect to 3D face recognition have been published. We describe the most recent approaches to the facial recognition challenge.

\section{3D Supported 2D Models}

Zhao and Chellappa proposed in [7] a shape-from-shading (SFS) method for preprocessing of 2D images. This SFS-based method used a depth map for generating synthetic frontal images. The The Linear Disrciminant Analysis (LDA) was applied to the synthetic images instead of the original images. The recognition rate increased with $4 \%$ when the synthetic images were used for LDA coding instead of the original images. Hu et al. proposed to use one neutral frontal image to first create a 3D model and from that create synthetic images under different poses, illuminations and expressions [8]. By applying LDA or Principal Component Analysis (PCA) to this 3D model instead of the $2 \mathrm{D}$ face images, the recognition rate increased with an average of $10 \%$ for the half-profile images. A similar idea was proposed earlier by Lee and Ranganath where they presented a combination of an edge model and color region model for face recognition after the synthetic images were created by a deformable 3D model [9]. Their method was tested on a dataset with 15 subjects and reached a recognition rate of $92.3 \%$ when 10 synthetic images per subject were used and $26.2 \%$ if one image for each subject was used.

\section{Surface Based Approaches}

\subsection{Local Methods}

Suikerbuik [11] proposed to use Gaussian curvatures to find 5 landmarks in a 3D model. He could find the correct landmark point with a maximal error of 4 $\mathrm{mm}$. Gordon proposed to use the Gaussian and cean curvature combined with depth maps to extract the regions of the eyes and the nose. He matched these regions to each other and reached a recognition rate of $97 \%$ on a dataset of 24 subjects [12]. Moreno et al. used both median and Gaussian curvature for the 
selection of 35 features in the face describing the nose and eye region [13]. The best recognition rate was reached on neutral faces with a recognition rate of $78 \%$.

$\mathrm{Xu}$ et al. proposed to use Gaussian-Hermite moments as local descriptors combined with a global mesh [14]. Their approach reached a recognition rate of $92 \%$ when tested on a dataset of 30 subjects. When the dataset was increased to 120 subjects, the recognition rate decreased to $69 \%$.

Chua et al. $[15,16]$ introduced point signatures to describe the 3D landmark. They used point signatures to describe the forehead, nose and eyes. Their method reached a recognition rate of $100 \%$ when tested on a dataset with 6 subjects. Wang et al. used the point signatures to describe local points on a face (landmarks). They tested their method on a dataset of 50 subjects and compared their results with the Gabor wavelet approach [17]. Their results showed that point signatures alone reached a recognition rate of $85 \%$ where the Gabor wavelets reached a recognition rate of $87 \%$. If both $2 \mathrm{D}$ and $3 \mathrm{D}$ landmarks were combined, they reached a recognition rate of $89 \%$. The authors remarked that these results could also be influenced by the number of landmarks used for face recognition, since for the point signatures 4 landmarks were used, for the Gabor wavelets 6 landmarks and for the combination of both 12 landmarks were used.

Douros and Buxton proposed the Gaussian Curvature to define quadratic patches to extract significant areas of the body. They claim that their method can be used for recognition of all kinds of 3D models [18]. Another local shape descriptor that was found to perform good on human bodies was the Paquet Shape Descriptor [19].

\subsection{Global Methods}

One global method on curvature was lately presented by Wong et al. [20]. The surface of a facial model was represented by an Extended Gaussian Image (EGI) to reduce the $3 \mathrm{D}$ face recognition problem to a $2 \mathrm{D}$ histogram comparison. The proposed measure was the multiple conditional probability mass function classifier (MCPMFC). Tested on a dataset of 5 subjects the MCPMFC has a recognition rate of $80.08 \%$ where a minimum distance classifier (MDC) reached a recognition rate of $67.40 \%$. However a test on synthetic data showed that for both methods the recognition rate decreased with $10 \%$ when the dataset was increased from 6 subjects to 21 subjects.

Papatheodorou and Rueckert proposed to use a combination of a 3D model and the texture of a face [21]. They also proposed some similarity measures for rigid alignment of two faces for 3D models and for 3D models combined with the texture. Their results showed an increase for frontal images when adding a texture to the model.

Beumier and Acheroy proposed to use vertical profiles of 3D models for face recognition. Their first attempt was based on three profiles of one face and had an error rate of $9.0 \%$ when it was tested on a dataset of 30 subjects [22]. In their second attempt they added grey value information to the matching process [23]. This attempt reduced the error rate to $2.5 \%$ when it was tested on the 
same database. Wu et al. proposed to perform 3D face recognition by extracting multiple horizontal profiles from the 3D model [24]. By matching these profiles to each other they reached an error rate between $1 \%$ and $5.5 \%$ tested on a database with 30 subjects.

\section{Template Matching Approaches}

Blanz, Vetter and Romdhani proposed to use a 3D morphable model for face recognition on 2D images [25-27]. With this method tested on a dataset of 68 subjects they reached a recognition rate of $99.8 \%$ for neutral frontal images and a recognition rate of $89 \%$ for profile images. Huang et al. added a component based approach to the morphable model [29] based on the approach of Heisele [28]. However, the recognition rate was for all approaches of the morphable model between the $75 \%$ and the $99 \%$.

Naftel et al. presented a method for automatically detecting landmarks in $3 \mathrm{D}$ models by using a stereo camera [30]. The landmarks were found on the 2D images by an ASM model. These landmark points were transformed to the 3D model by the stereo camera algorithm. This algorithm was correct in $80 \%$ of all cases when tested on a dataset of 25 subjects.

A similar idea was proposed by Ansari and Abdel-Mottaleb [31]. They used the CANDIDE-3 model [32] for face recognition. Based on a stereo images landmark points around the eyes, nose and mouth were extracted from the 2D images and converted to 3D landmark points. A 3D model was created by transforming the CANDIDE-3 generic face to match the landmark points. The eyes, nose and mouth of the 3D model were separately matched during the face recognition. Their method achieved a recognition rate of $96.2 \%$ using a database of 26 subjects. Lu et al. had used the generic head from Terzopoulos and Waters [33] which they adapted for each subject based on manually placed feature points in the facial image [34]. Afterwards the models were matched based on PCA. This method was tested on frontal images and returns in $97 \%$ of all cases the correct face within the best 5 matches.

\section{Other Approaches}

The original principal component method for 3D facial models was implemented by Mavridis et al. for the European project HiScore [35]. Chang et al. had compared the performance of $3 \mathrm{D}$ eigenfaces and $2 \mathrm{D}$ eigenfaces of neutral frontal faces on a dataset of 166 subjects [36]. They found no real difference in performance for the 2D eigenfaces and 3D eigenfaces. However, a combination of both dimensionalities scored best of all with a recognition rate of $82.8 \%$. Xu et al. proposed to use 3D eigenfaces with nearest neighbor and k-nearest neighbors as classifiers [37]. Their approach reached a recognition rate around the $70 \%$ when tested on a dataset of 120 subjects.

Bronstein et al. had proposed to transform the 3D models to a canonical form before applying the eigenface method to it [38]. They claimed that their 
method could discriminate between identical twins and was insensitive for facial expressions, although no recognition rates were given.

Tsalakanidou et al. proposed to combine depth maps with intensity images. In their first attempt they used eigenfaces for the face recognition and his results showed a recognition rate of $99 \%$ for a combination of both on a database of 40 subjects [39]. In a second attempt embedded hidden markov models were used instead of eigenfaces to combine the depth images and intensity images [40]. This approach had an error rate between the $7 \%$ and $9 \%$.

\section{Discussion and Conclusion}

It is hard to compare the results of different methods to each other since the experiments presented in literature are mostly performed under different conditions on different sized datasets. For example one method was tested on neutral frontal images and had a high recognition rate, while another method was tested on noisy images with different facial expressions or head poses and had a low error rate.

Some authors presented combinations of different approaches for a face recognition method and these performed all a little better than the separate methods. But besides recognition rate, the error rate and computational costs are important, too. If the error rate decreases significantly, while the recognition rate increases only a little bit, the combined method is still preferred. But, if the computational costs increase a lot, calculation times could become prohibitive for practical applications.

Most interesting for this survey were the studies that presented method comparisons, like [41-43]. Phillips et al. [6] present comparison studies performed on the FERET database. The latest FERET test performed on different algorithms was presented in 2000 [44]. An important conclusion from this survey was that the recognition rates of all methods improved over the years. The dynamic graph matching approach of Wistkott et al. [17] had the best overall performance on identification. For face verification the combination of PCA and LDA presented by Zhao et al. [46] performed best.

In table 1 a summary is given for the most important and successful 2D and $3 \mathrm{D}$ face recognition methods. One can see that the 3D face recognition approaches are still tested on very small datasets. However, the datasets are increasing during the years since better acquisition materials become available. By increasing a dataset, however, the recognition rate will decrease. So the algorithms must be adjusted and improved before they will be able to handle large datasets with the same recognition performance. Another disadvantage of most presented 3D face recognition methods is that most algorithms still treat the human face as a rigid object. This means that the methods aren't capable of handling facial expressions. In contrast to 3D face recognition algorithms, most $2 \mathrm{D}$ face recognition algorithms are already tested on large datasets and are able to handle the size of the data tolerable well. The last few years more and more 2D face recognition algorithms are improved and tested on less perfect images, 
like noisy images, half profile images, occlusion images, images with different facial expressions, et cetera. Although not single algorithm can be assumed to handle the difficult images good enough, an increasing line in performance can be found.

Although 2D face recognition still seems to outperform the 3D face recognition methods, it is expected that in the near future $3 \mathrm{D}$ face recognition methods outperform 2D methods. 3D models hold more information of the face, like surface information, that can be used for face recognition or subject discrimination. Another major advantage is that $3 \mathrm{D}$ face recognition is pose invariant. Therefore, $3 \mathrm{D}$ face recognition is still a challenging but very promising research area.

\section{References}

1. Bledsoe, W.W.: The Model Method in Facial Recognition. Technical Report PRI-15. Panoramic Recsearch Inc. Califormia (1966)

2. Bledsoe,W.W.: Man-Machine Facial Recognition: Report on a Large-Scale Experiment. Technical Report PRI-22. Panoramic Recsearch Inc. Califormia (1966)

3. Samal, A., Iyengar,P.A.: Automatic Recognition and Analysis of Human Faces and Facial Expressions. Pattern Recognition. 25 (1992) 65-77

4. Chellappa, R., Wilson, C.L., Sirohey. S.: Human and Machine Recognition of Faces: A survey. Proceedings of the IEEE, 83 (1995) 705-740

5. Zhao, W.Y., Chellappa, R., Phillips, P.J., Rosenfeld, A.: Face Recognition: A Literature Survey. ACM Computing Surveys. 35 (2003) 399-458

6. Phillips, P.J., Grother,R., Micheals, R.J., Blackburn, D.M., Tabassi, E., Bone, M.: Face Recognition Vendor test 2002. "http://www.frvt.org/" (December 2004)

7. Zhao,W.Y., Chellappa,R.: SFS Based View Synthesis for Robust Face Recognition. Proceedings of the IEEE International Automatic Face and Gesture Recognition (2000) 285-292

8. Hu,Y., Jiang, D., Yan, S.,Zhang, L., Zhang, H.: Automatic 3D reconstruction for Face Recognition. Proceedings of the IEEE International Conference on Automatic Face and Gesture Recognition. (2000) 843-850

9. Lee, C.H., Park, S.W.,Chang, W., Park, J.W.: Improving the performance of MultiClass SVMs in face recognition with nearest neighbour rule. Proceedings of the IEEE International Conference on Tools with Artificial Intelligence. (2003) 411-415

10. Bowyer, K.W., Chang, K., Flynn, P.J.: A survey of approaches to three-dimensional face recognition. International Conference of Pattern Recognition, Vol I. (2004) 358361

11. Suikerbuik, C.A.M., Tangelder, J.W.H., Daanen, H.A.M., Oudenhuijzen, A.J.K.: Automatic feature detection in 3D human body scans. Proceedings of the conference "SAE Digital Human Modelling for Design and Engineering. (2004)

12. Gordon, G.G.: Face Recognition Based on Depth Maps and Surface Curvature. Proceedings of the SPIE, Geometric Methods in Computer Vision, Vol. 1570. (1991) $108-110$

13. Moreno, A.B., Sánchez, A., Vélez, J.F., Díaz, F.J.: Face Recognition using 3D Surface-Extracted Descriptors. Proceedings of the Irish Machine Vision and Image Processing Conference. (2003)

14. Xu, C., Wang, Y., Tan, T., Quan, L.: Automatic 3D Face recognition combining global geometric features with local shape variation information. Proceedings of the 
IEEE International Conference on Automatic Face and Gesture Recognition. (2004) 302-307

15. Chua, C.S., Jarvis, R.: Point Signatures: A New Representation for 3D Object Recognition. International Journal on Cumputer Vision. 25 (1997) 63-85

16. Chua, C.S., Han, F., Ho, Y.K.: 3D human face recognition using point signature. Proceedings of the IEEE International Conference on Automatic Face and Gesture Recognition. (2000) 233-238

17. Wiskott, L., Fellous, J.M., kruger, N., van der Malsburg, C.: Face Recognition by Elastic Bunch Graph Matching. IEEE Transactions on Pattern Analysis and Machine Intelligence. 19 (1997) 775-779

18. Douros, I., Buxton, B.F.: Three-Dimensional Surface Curvature Estimation using Quadric Surface Patches. Proceedings of the Scanning 2002 Conference. (2002)

19. Robinette, K.M., An Alternative 3D descriptor for database mining. Proceedings of the Digital Human Modelling Conference. (2004)

20. Wong, H.S., Chueng, K.K.T., Ip, H.H.S.: 3D head model classification by evolutionary optimization of the extended Gaussian image representation. Pattern Recognition. 37 (2004) 2307-2322

21. Papatheodorou, T., Rueckert, D.: Evaluation of automatic 4D Face recognition using surface and texture registration. Proceedings of the IEEE International Conference in Automatic Face and Gesture Recognition. (2004) 321-326

22. Beumier, C., Acheroy, M.: Automatic 3D face authentication. Image and Vision Computing. 18 (2000) 315-321

23. Beumier, C., Acheroy, M.: Face verification from 3D and grey level clues. Pattern Recognition Letters. 22 (2001) 1321-1329

24. Wu, Y., Pan, G., Wu, Z.: Face Authentication Based on Multiple Profiles Extracted from range data. Proceedings of the Audio- and Video-Based Biometric Person Authentication, Vol. 2688. (2003) 515-522

25. Blanz, V., Romdhani, S., Vetter, T.: Face Identification across different poses and illuminations with a 3D morphable model. Proceedings of the IEEE International Automatic Face and Gesture Recognition. (2002)

26. Blanz, V., Vetter, T.: Face Recognition Based on Fitting a 3D Morphable Model. IEEE Transactions on Pattern Analysis and Machine Intelligence. 25 (2003)

27. Romdhani, S., Vetter, T.: Efficient, Robust and Accurate Fitting of a 3D Morphable Model. Proceedings of the European Conference on Computer Vision. (2003)

28. Heisele, B., Koshizen, T.: Components for Face Recognition. Proceedings of the Audio- and Video-Based Biometric Person Authentication, Vol 2688. (2003) 153-159

29. Huang, J., Heisele, B., Blanz, V.: Component-Based Face Recognition with 3D Morphable Models. Proceedings of the Audio- and Video-Based Biometric Person Authentication, Vol 2688. (2003) 27-34

30. Naftal, A.J., Mao, Z., Trenouth, M.J.: Stereo-assisted landmark detection for the analysis of 3D facial shape changes. Technical Report TRS-2002-007. Deprtment of Computation UMIST, Manchester. (2002)

31. Ansari, A., Abdel-Mottaleb, M.: 3D Face modeling using two views and a generic face model with application to 3D face recognition. Proceedings of the IEEE Conference on Advanced Video and Signal Based Surveillance. (2003) 37-44

32. Ahlberg, J.: CANDIDE-3 - an updated parameterized face. Technical Report LiTHISY-R-2326. Dept. of Electrical Engineering, Linkping University. (2001)

33. Terzopoulos, D., Waters, K.: Analysis and Synthesis of Facial Image Sequences Using Physical and Anatomical Models. IEEE Transactions on Pattern Analysis and Machine Intelligence. 15 (1993) 569-579 
34. Lu, X., Hsu, R., Jain, A., Kamgar-Parsi, B.: Face Recognition with 3D ModelBased Synthesis. Proceedings of the International Conference on Biometric Authentication. (2004) 139-146

35. Mavridis, N., Tsalakanidou, F., Pantazis, D., Malasiotis, S., Strintzis, M.: The HISCORE face recognition application: Affordable desktop face recognition based on a novel 3D camera. Proceedings of the International Conference on Augmented Virtual Environments and 3D Images. (2001) URL: http://uranus.ee.auth.gr/hiscore

36. Chang, K.I., Bowyer, K.W., Flynn, P.J.: Multi-Modal 2D and 3D Biometrics for Face Recognition. Proceedings of the IEEE International Workshop on Analysis and Modeling Journal on Computer Vision. (2003)

37. Xu, C., Wang, Y., Tan, T., Quan, L.: A New Attempt to Face Recognition Using Eigenfaces. Proceedings of the Asian Conference on Computer Vision. 2 (2004) 884889

38. Bronstein, A.M., Bronstein, M.M., Kimmel, R.: Expression-Invariant 3D Face Recognition. Audio- and Video-Based Biometric Person Authentification, Vol 2688. (2003) 62-70

39. Tsalakanidou, F., Tzovaras, D., Strintzis, M.G.: Use of depth and colour eigenfaces for face recognition. Pattern Recognition Letters. 24 (2003) 1427-1435

40. Tsalakanidou, F., Malassiotis, S., Strintzis, M.G.: Integration of 2D and 3D images for Enhanced Face Authentication. Proceedings of the IEEE International Conference in Automatic Face and Gesture Recognition. (2004) 266-271

41. Navarrete, P., Ruiz-del-Solar, J.: Comparative Study between different eigenspacebased approaches for face recognition. AFSS International Conference on Fuzzy Systems. (2002) 178-187

42. Jonsson, K., Kittler, J., Matas, J.: Support Vector Machines for face authentication. Image and Vision Computing. 20 (2002) 369-375

43. Sadeghi, M., Kittler, J., Kostin, A., Messer, K.: A comparative study of automatic face verification algorithms on the BANCA database. Proceedings of the Audioand Video-Based Biometric Person Authentication, Vol 2688. (2003) 35-43

44. Phillips, P.J., Moon, H., Rizvi, S.A., Rauss, P.J.: The FERET Evaluation Methodology for Face-Recognition Algorithms. IEEE Transactions on Pattern Analysis and Machine Intelligence. 22 (2000) 1090-1104

45. Wiskott, L.: Phantom Faces for Face Analysis. Proceedings of the Joint Symposium on Neural Computation. (1996) 46-52

46. Zhao, W.Y., Krishnaswamy, A., Chellappa, R.: Discriminant Analysis of Principal Components for Face Recognition. Proceedings of the International Conference on Automatic Face and Gesture Recognition. (1998) 336-341

47. Moghaddam, B., Jebera, T., Pentland, A.P.: Bayesian face recognition. Pattern Recognition. 33 (2000) 1771-1782

48. Lanitis, A., Taylor, C.J., Cootes, T.F.: An automatic face identification system using flexible appearance models. Image and Vision Computing. 13 (1995) 393-401

49. Pan, Z., Healey, G., Prasad, M., Tromberg, B.: Face Recognition in Hyperspectral Images. IEEE Transactions on Pattern Analysis and Machine Intelligence. 25 (2003) $1552-1560$ 


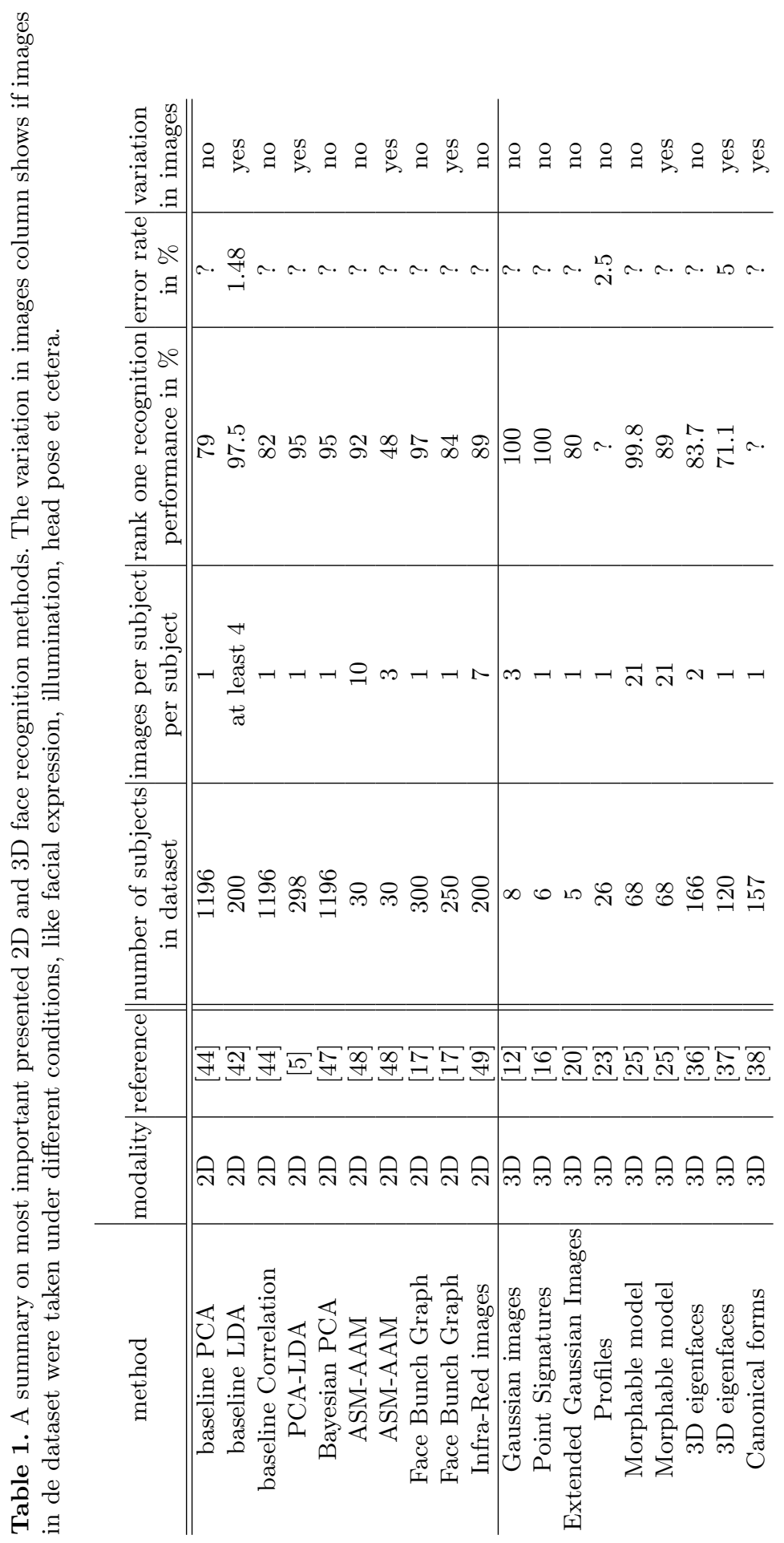

